\title{
Intracellular nanomanipulation by a photonic-force microscope with real-time acquisition of a 3D stiffness matrix
}

\author{
E Bertseva ${ }^{1}$, A S G Singh ${ }^{2}$, J Lekki $^{3}$, P Thévenaz $^{2}$, M Lekka $^{3}$, \\ S Jeney ${ }^{1}$, G Gremaud ${ }^{1}$, S Puttini ${ }^{1}$, W Nowak ${ }^{4}$, G Dietler ${ }^{1}$, L Forró ${ }^{1}$, \\ M Unser ${ }^{2}$ and A J Kulik ${ }^{1}$ \\ ${ }^{1}$ EPFL/SB/IPMC/LNNME, Station 3, CH-1015 Lausanne VD, Switzerland \\ ${ }^{2}$ EPFL/STI/IMT/LIB, Station 17, CH-1015 Lausanne VD, Switzerland \\ ${ }^{3}$ Institute of Nuclear Physics, Polish Academy of Sciences, Radzikowskiego 152, \\ PL-31-342 Krakow, Poland \\ ${ }^{4}$ Institute of Physics, Nicolaus Copernicus University, ulica Grudziadzka 5, \\ PL-87-100 Torun, Poland \\ E-mail: andrzej.kulik@epfl.ch
}

Received 11 March 2009

Published 24 June 2009

Online at stacks.iop.org/Nano/20/285709

\begin{abstract}
A traditional photonic-force microscope (PFM) results in huge sets of data, which requires tedious numerical analysis. In this paper, we propose instead an analog signal processor to attain real-time capabilities while retaining the richness of the traditional PFM data. Our system is devoted to intracellular measurements and is fully interactive through the use of a haptic joystick. Using our specialized analog hardware along with a dedicated algorithm, we can extract the full 3D stiffness matrix of the optical trap in real time, including the off-diagonal cross-terms. Our system is also capable of simultaneously recording data for subsequent offline analysis. This allows us to check that a good correlation exists between the classical analysis of stiffness and our real-time measurements. We monitor the PFM beads using an optical microscope. The force-feedback mechanism of the haptic joystick helps us in interactively guiding the bead inside living cells and collecting information from its (possibly anisotropic) environment. The instantaneous stiffness measurements are also displayed in real time on a graphical user interface. The whole system has been built and is operational; here we present early results that confirm the consistency of the real-time measurements with offline computations.
\end{abstract}

(Some figures in this article are in colour only in the electronic version)

\section{Introduction}

A photonic-force microscope (PFM) is an instrument that uses a focused laser beam to trap a micrometer-sized bead within some medium. Random thermal fluctuations act upon this bead so that it acquires a Brownian motion, and the position of the bead is recorded through time. The appropriate statistical analysis of the recorded path provides insights on the properties of the medium at the location of the bead [1-3].

In this paper, we propose a human-controlled instrument that combines (1) a PFM; (2) actuators to move the bead relative to the medium within a much larger range than that achieved by the thermal fluctuations; (3) a haptic joystick to interactively drive these actuators and to let the bead explore the medium; (4) a real-time determination of the local properties of the medium at the location of the bead; (5) a force-feedback mechanism that acts on the haptic joystick according to the properties of the medium and (6) a graphical monitoring of the whole process. The actuators control the macro-position of the bead along the three orthogonal directions of space. Moreover, we take advantage of an ultra-fast quadrant photodiode (QPD) to record, also in three 


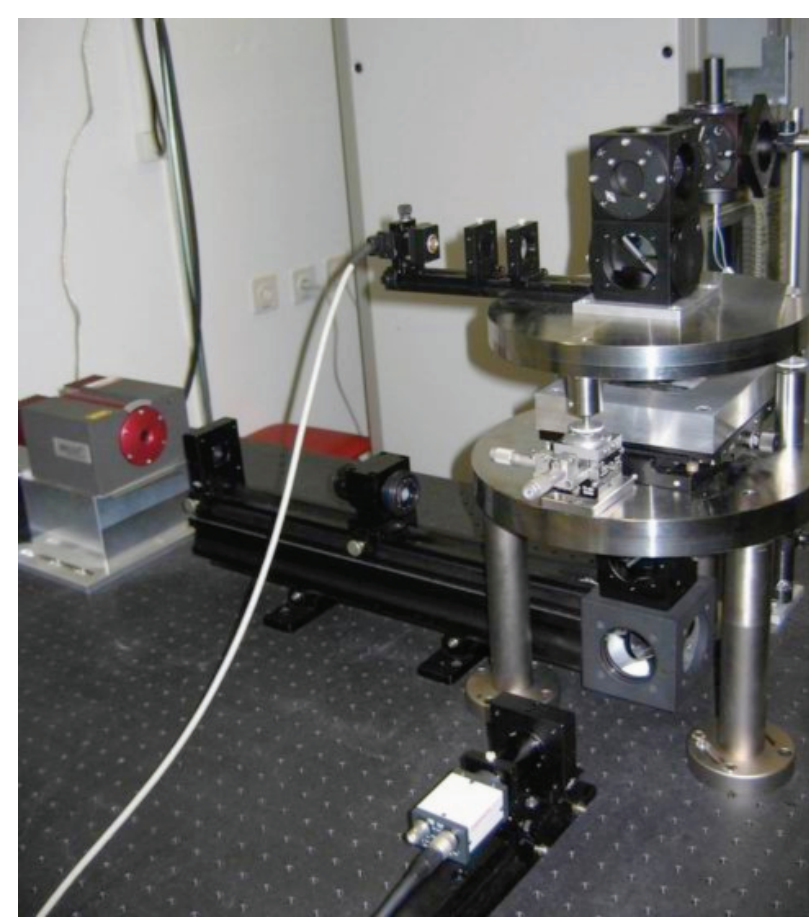

Figure 1. Photograph of our PFM set-up.

dimensions, the micro-fluctuations of the position of the bead; the resulting path is made available for offline processing. In addition, we determine in real time the local properties of the medium thanks to a combination of hardware and software, which is a major innovation of this paper.

Not only do thermally induced fluctuations of the position of the trapped bead provide access to the viscoelastic properties of aqueous environments [1], they also provide a valuable tool to measure the mechanical properties of single motor molecules with high resolution [4]. Moreover, PFM can be used to image in 3D the topology of a polymer network [5]. Unfortunately, these analyses require the processing of a large amount of data. Until now, the calculations have been done offline-which is extremely time-consuming — and the results have not been available for interactive reaction by the scientist who is conducting the experiment. By contrast, our system allows for real-time manipulation of the bead inserted inside a living cell, for online data processing and computation of the $3 \mathrm{D}$ matrix that models the local intracellular stiffness, for immediate and intuitive visualization of this quantity, and for using it to deliver an instantaneous force-based haptic feedback to the user.

In addition to its real-time performance, our system logs data in the form of sequential records containing every physical information collected in the experiment: a time stamp, the position of the probe, the raw high-speed PFM readout and its low-speed hardware-processed counterpart. The latter contains all the information needed for real-time operations such as online stiffness determination, haptic loop and visualization tasks, while the collected high-speed signal provides data with nanometer spatial resolution and microsecond temporal resolution, which are suitable to more traditional offline PFM analyses $[6,7]$. By making both high-speed and low-speed data

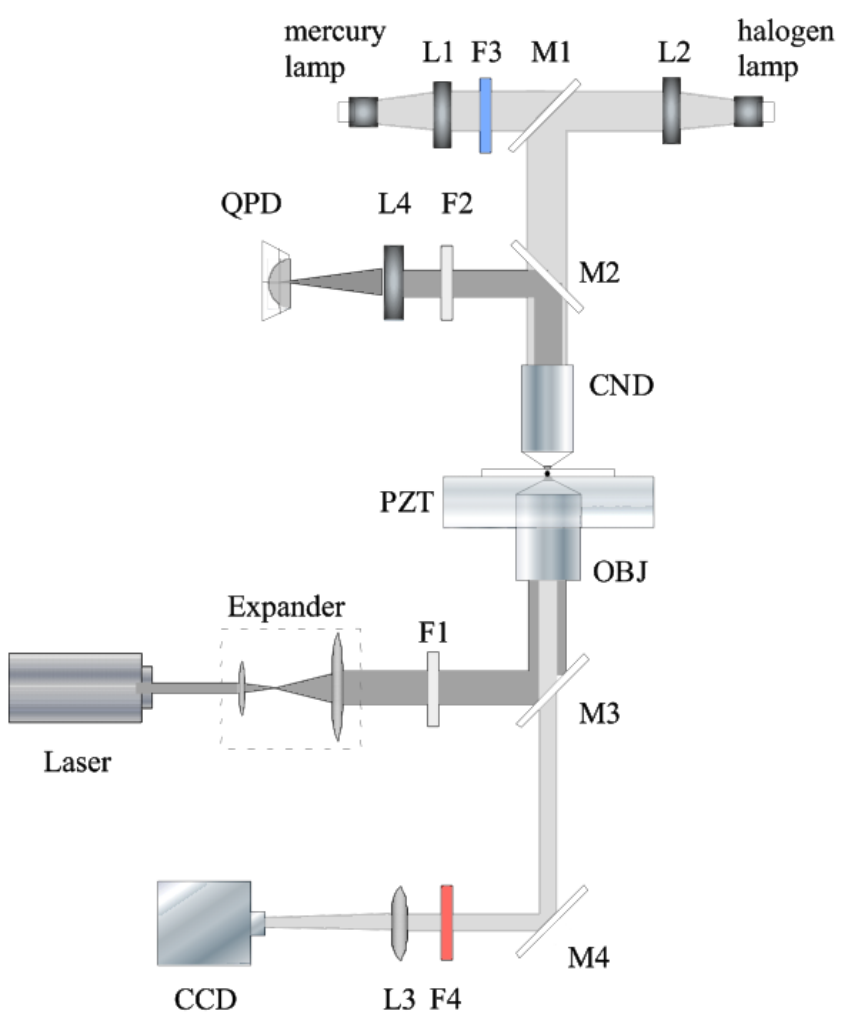

Figure 2. Principal scheme of our PFM. Laser: Nd:YAG laser; L1-L4: lenses; M1, M4: aluminum mirrors; M2, M3: dichroic mirrors; F1, F2: neutral filters; F3, F4: bandpass filters; OBJ: objective; CND: condenser; PZT: piezoelectric motorized stage; QPD: quadrant photodiode; CCD: camera.

available, we allow one to easily single out the most interesting parts of the experiment, thus focusing on the relevant data and therefore shortening the duration of any subsequent offline analysis.

\section{Experimental set-up}

Some features of our PFM system are very similar to the one described in detail in [8]. We provide in figure 1 its photograph and in figure 2 its general scheme. This set-up is centered around an Nd:YAG laser that was specially designed to achieve high-stability operation; its wavelength is $1064 \mathrm{~nm}$ and it has a maximum power of $2 \mathrm{~W}$ (MEPHISTO Innolight, $\mathrm{GmbH}$ ).

The laser beam passes through a $(10 \times)$ beam expander, is attenuated by the neutral filter F1 and is directed by the dichroic mirror M3 to a $(60 \times)$ water-immersion objective OBJ (Olympus UPlanApo/IR, NA = 1.2). The sample is placed above the objective on a piezoelectric translation stage PZT that allows for movements in the two horizontal and in the vertical directions. The laser light is collected by a $(63 \times)$ objective CND (Carl Zeiss Achroplan IR with NA = 0.9), which is also of a water-immersion type. The lens L4, with a focal length of $50 \mathrm{~mm}$, projects the image of the back-focalplane of the condenser onto the InGaAs quadrant photodiode QPD where interference patterns are collected (IGA-030-QD, Electro-Optical Systems Inc.). The QPD signals are fed to a custom-built preamplifier (Öffner MSR-Technik, Germany) 
which provides one signal that is proportional to the total light intensity, and two signals that are differential. We use these signals to determine the displacement of the bead $[9,10]$; in section 3.3, we shall see in more detail how we harness them.

The trapping (infrared) and the observation (visible) light paths are split by the dichroic mirrors M2 and M3. The infrared part is responsible for bead trapping and position detection, while the visible part enables direct observation and video recording. The observation part consists of a fluorescence optical microscope and a high-quality CCD camera. The microscope can be operated with the aid of either the halogen lamp in normal mode or the mercury lamp in fluorescence mode. We tune the orientation of the rotating mirror M1 to select one of these two sources. Finally, we use a CCD camera (Marlin F-131, $(1280 \times 1024)$ elements) to acquire images and videos, and to record them thanks to the commercial software (MediaWorks 6.2).

The filters for fluorescence are adapted to the beads we used (Fluoresbrite ${ }^{\circledR}$ YG carboxylate Microspheres, $0.30 \mu \mathrm{m}$ in diameter). We chose the following passbands: excitation 430$470 \mathrm{~nm}$, emission > $500 \mathrm{~nm}$. Non-fluorescent polystyrene beads of various sizes (Bangs Laboratories) have been used too.

The position of the piezoelectric table is driven by a 3D haptic joystick, which enforces that the medium being sampled moves relative to the optical trap. Meanwhile, the data collected by the QPD are analyzed, and the stiffness of the medium surrounding the bead is converted into forces exerted by the joystick in a feedback loop that takes into account the counteracting drag forces proportional to the velocity of the table. In this way, the operator gets to 'feel' local environmental properties. We give in section 4 a more detailed description of this nanomanipulator part of our system.

\section{Data processing}

\subsection{The Langevin process}

The working principle of a photonic-force microscope is that a small bead is buffeted by random thermal fluctuations of the medium in which it is placed, and at the same time it is attracted to the center of the optical trap induced by the focused beam of a laser. If we let the instantaneous position of the bead be described by $\mathbf{x}$ and its velocity and acceleration by $\dot{\mathbf{x}}$ and $\ddot{\mathbf{x}}$, respectively, then a convenient description of the dynamics of the system is given by the Langevin equation

$$
\mathbf{M} \ddot{\mathbf{x}}+\Gamma \dot{\mathbf{x}}+\mathbf{K} \mathbf{x}=\mathbf{F} \mathbf{1}
$$

There, $\mathbf{M}$ is the mass matrix of the bead, $\boldsymbol{\Gamma}$ is a friction matrix related to the medium, $\mathbf{K}$ is the stiffness matrix of the optical trap and the product of the matrix $\mathbf{F}$ with the column vector $\mathbf{1}=(1,1,1)$ represents the contribution of the driving force.

In all generality, this system has no less than 36 free parameters (the elements of the four $(3 \times 3)$ matrices $\mathbf{M}, \boldsymbol{\Gamma}, \mathbf{K}$ and $\mathbf{F}$ ); moreover, the stochastic nature of $\mathbf{F}$ makes it difficult to handle. As our goal is to fit the parameters of the Langevin model to the experimental path taken by the bead, we now make a few reasonable simplifications to make the problem tractable.
- We assume that all matrices, except $\mathbf{F}$, are symmetric. This said, we retain off-diagonal terms, which is one important contribution of our method.

- The experimental conditions are such that the bead is trapped. Therefore, (1) describes a stationary system, which allows us to escape several difficult mathematical considerations on the stochastic nature of the process.

- We assume that the driving force $\mathbf{F}$ can be modeled as zero-mean white noise; moreover, $\mathbf{F}$ depends on the time $t$ alone but not on $\mathbf{x}$, while (F $\mathbf{1}$ ) varies much more rapidly than $\mathbf{x}$.

Finally, our last hypothesis is known as the fluctuationdissipation relation. It can be stated as

$$
\left\{\begin{array}{l}
\mathrm{E}\left\{F_{i j}\left(t_{1}\right) F_{i j}\left(t_{2}\right)\right\}=\mathrm{E}\left\{F_{i j}\left(t_{1}\right) F_{j i}\left(t_{2}\right)\right\} \\
\mathrm{E}\left\{F_{i j}\left(t_{1}\right) F_{i j}\left(t_{2}\right)\right\}=2 k_{\mathrm{B}} T \gamma_{i j} \delta\left(t_{1}-t_{2}\right) \\
\mathrm{E}\left\{F_{i j}\left(t_{1}\right) F_{k l}\left(t_{2}\right)\right\}=0,
\end{array}\right.
$$

where $\mathrm{E}$ is the expectation operator and where $k_{\mathrm{B}}$ and $T$ are Boltzmann's constant and the temperature of the medium surrounding the bead, respectively. Under these conditions, a classical result from [11] is that the random position $\mathbf{x}$ that satisfies (1) corresponds to $\mathrm{E}\{\mathbf{x}\}=\mathbf{x}_{0}$, where $\mathbf{x}_{0}$ does not depend on time. Moreover, $\mathbf{x}$ follows a Gaussian distribution given by

$$
p(\mathbf{x})=\frac{1}{\sqrt{(2 \pi)^{3}|\operatorname{det}(\mathbf{C})|}} \mathrm{e}^{-\frac{1}{2} \mathbf{x}^{\top} \mathbf{C}^{-1} \mathbf{x}},
$$

where the autocovariance of the bead displacement is found to be

$$
\mathbf{C}=k_{\mathrm{B}} T \mathbf{K}^{-1} \text {. }
$$

\subsection{Traditional stiffness matrix}

In an isotropic medium, and when the optical axis is aligned with the system of coordinates, we can assume $\mathbf{C}$ and $\mathbf{K}$ in (2) to be diagonal matrices. Then, following [12], we can estimate the $i$ th diagonal component of the stiffness matrix of the optical trap as given by $\tilde{k}_{i}=\frac{k_{\mathrm{B}} T}{c_{i i}}$. If, in addition, we build an estimated position $\tilde{\mathbf{x}}$ of the bead by applying a simple linear-conversion factor to the signal $\mathbf{y}$ returned by the QPD, so that $\tilde{x}_{i}=y_{i} / \beta_{i}$, then the stiffness becomes

$$
\tilde{k}_{i}=\frac{\beta_{i}^{2} k_{\mathrm{B}} T}{\sigma_{i}^{2}},
$$

where $\sigma_{i}=\sigma_{i i}$, with $\sigma_{i j}^{2}=[\Sigma]_{i j}$ the $i j$ th element of the autocovariance matrix $\Sigma$ of $\mathbf{y}$. In practice, to obtain the coefficients $\left(\beta_{1}, \beta_{2}, \beta_{3}\right)$, we turn to a calibration method that involves the detection of a plateau in the spectrum of the measurements [13].

While simple, the traditional procedure to obtain the stiffness of the trap that we have just exposed, unfortunately, ignores many effects such as nonlinearities of the detector and is incompatible with the potential existence of crossterms. Moreover, it does not take into account artificial correlations introduced by the QPD detector. In this paper instead, we propose an improved estimate of $\mathbf{K}$ that takes non-diagonal terms into consideration and that removes the 


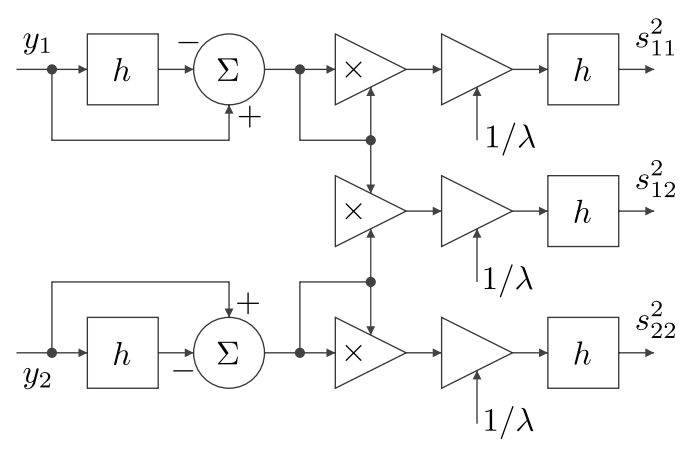

Figure 3. Schematics of the analog processor used to get $\mathbf{S}$ given $\mathbf{y}$. In the interest of space, only three out of the six independent elements of $\mathbf{S}$ are shown here.

artificial correlations introduced by the QPD detector. Our estimate is based on the statistical analysis of a collection of measured signals $\mathbf{y}$. It allows us to ascertain the stiffness of the trap-or the stiffness of the medium surrounding the bead, depending on their relative impact.

\subsection{Hardware implementation}

While our system is fast enough to sample the three components of $\mathbf{y}$ at a high sampling rate (typically, $1 \mathrm{MHz}$ ), we are unable to perform their further processing in real time; instead, we simply store them for later inspection. Because it would be difficult to obtain $\mathbf{K}^{-1}$ with online numerical computations, we have built a hardware device to provide us with an estimate of the autocovariance $\mathbf{C}$ over a sliding time window.

Since $\mathbf{C}=\mathrm{E}\left\{(\mathbf{x}-\mathrm{E}\{\mathbf{x}\})(\mathbf{x}-\mathrm{E}\{\mathbf{x}\})^{\top}\right\}$, we need practical realizations of the expectation process $\mathrm{E}$, which we obtain thanks to analog low-pass filters. We therefore get a timevarying access to the quantity $\mathrm{E}\{\mathbf{x}\}$ by creating the signal $\overline{\mathbf{y}}(t)=\int_{-\infty}^{t} \mathbf{y}(\tau) h(t-\tau) \mathrm{d} \tau$, where $h$ is the impulse response of the filter. We have chosen to build $h$ as the cascade of two standard first-order RC (active) filters, which ensures that $h$ is causal and nonnegative, along with $\int_{-\infty}^{\infty} h(\tau) \mathrm{d} \tau=1$. We have set the cutoff frequency of $h$ to $500 \mathrm{~Hz}$.

The signal $\left(y_{i}-\bar{y}_{i}\right)$ is then multiplied by $\left(y_{j}-\bar{y}_{j}\right)$. As this multiplication is realized in analog fashion, a scaling factor $\lambda^{-1}$ is required to homogenize the physical units. In our case, this factor is such that multiplying together two signals of $1 \mathrm{~V}$ each results in an output signal of $0.1 \mathrm{~V}$, so that $\lambda=10$. The outermost expectation operator defining $\mathbf{C}$ is finally realized as yet another low-pass digital filter, with identical order, make and cutoff frequency. This yields the components $s_{i j}$ of the scatter matrix $\mathbf{S}$ that estimates, up to the multiplicative factor $\lambda$, the autocovariance matrix $\boldsymbol{\Sigma}$ of the measurements $\mathbf{y}$. We give in figure 3 a partial schematic diagram of our device; the components that are missing from this figure are realized similarly, up to a permutation of the indices.

Because $h$ is a low-pass filter, we can sample $s_{i j}^{2}=\sigma_{i j}^{2} / \lambda$ at a much lower rate $(1 \mathrm{kHz}$ in our current system) than we sample y. In this way, we can now process the data numerically in real time. For example, we can reduce the influence of noise by computing a time average of the slow signal. As we now proceed numerically rather than in analog fashion, we avoid any problem of stability.

\subsection{Proposed stiffness matrix}

Taking into account artificial correlations introduced by the QPD (derivations not shown), it is possible to cancel them and to relate the inverse $\mathbf{K}^{-1}$ of the stiffness of the trap to the scatter matrix $\mathbf{S}=\frac{1}{\lambda} \mathbf{\Sigma}$ that we obtained thanks to the hardware we just described in section 3.3. The general strategy is to use (2) to get $\mathbf{K}$ from $\mathbf{C}$, to get an estimate of $\mathbf{C}$ from $\boldsymbol{\Sigma}$ thanks to $\beta_{i}$, and to get $\boldsymbol{\Sigma}$ from $\mathbf{S}$ thanks to $\lambda$. We have that

$$
\begin{aligned}
& {\left[\mathbf{K}^{-1}\right]_{11}=\frac{\lambda}{k_{\mathrm{B}} T}\left(\frac{s_{11}^{2}}{\beta_{1}^{2}}-\frac{\lambda}{\bar{y}_{3}^{2}} \frac{s_{13}^{4}}{\beta_{1}^{2}}\right)\left(1+\frac{\lambda}{\bar{y}_{3}^{2}} s_{33}^{2}\right)^{-1}} \\
& {\left[\mathbf{K}^{-1}\right]_{12}=\frac{\lambda}{k_{\mathrm{B}} T}\left(\frac{s_{12}^{2}}{\beta_{1} \beta_{2}}-\frac{\lambda}{\bar{y}_{3}^{2}} \frac{s_{13}^{2} s_{23}^{2}}{\beta_{1} \beta_{2}}\right)\left(1+\frac{\lambda}{\bar{y}_{3}^{2}} s_{33}^{2}\right)^{-1}} \\
& {\left[\mathbf{K}^{-1}\right]_{13}=\frac{\lambda}{k_{\mathrm{B}} T} \frac{s_{13}^{2}}{\beta_{1} \beta_{3}}} \\
& {\left[\mathbf{K}^{-1}\right]_{22}=\frac{\lambda}{k_{\mathrm{B}} T}\left(\frac{s_{22}^{2}}{\beta_{2}^{2}}-\frac{\lambda}{\bar{y}_{3}^{2}} \frac{s_{23}^{4}}{\beta_{2}^{2}}\right)\left(1+\frac{\lambda}{\bar{y}_{3}^{2}} s_{33}^{2}\right)^{-1}} \\
& {\left[\mathbf{K}^{-1}\right]_{23}=\frac{\lambda}{k_{\mathrm{B}} T} \frac{s_{23}^{2}}{\beta_{2} \beta_{3}}} \\
& {\left[\mathbf{K}^{-1}\right]_{33}=\frac{\lambda}{k_{\mathrm{B}} T} \frac{s_{33}^{2}}{\beta_{3}^{2}}}
\end{aligned}
$$

where the quantities $\beta_{i}$ are identical to those found in section 3.2, but are now seen as mere unit-conversion factors.

To clarify how our approach relates to the traditional one, we want now to compare (4) to (3). We first observe that $\bar{y}_{3}$ measures the total intensity reaching the QPD; at the limit of the simplifying assumption that this intensity is strong, we can make the hypothesis that $\bar{y}_{3} \rightarrow \infty$. Then, we observe that $\tilde{k}_{i}=\left[\mathbf{K}^{-1}\right]_{i i}^{-1} \neq[\mathbf{K}]_{i i}$. But if, in addition, we assume that $\mathbf{K}$ is diagonal, then we finally have that $\tilde{k}_{i}=[\mathbf{K}]_{i i}$. Consequently, (4) is equivalent to (3) for bright illumination, isotropic medium and when the system of coordinates is aligned with the optical axis. Relaxing these restrictions yields the general form of our stiffness matrix.

To summarize, the method that we propose to obtain the stiffness of the optical trap results in a symmetric matrix $\mathbf{K}$ that takes cross-terms into account. As our detector is a quadrant photodiode, it creates artificial correlations between the measurements; we suggest a computational procedure to compensate for them. We have built an analog processor to handle the primary high-bandwidth signal. It generates a lowpass signal that we further process numerically. Our set-up allows us to attain real-time performance, which is a necessary requirement of the haptic loop.

\section{Overview of the nanomanipulator}

\subsection{Modules}

The nanomanipulator device has been built from the six following main modules (see figure 4): 


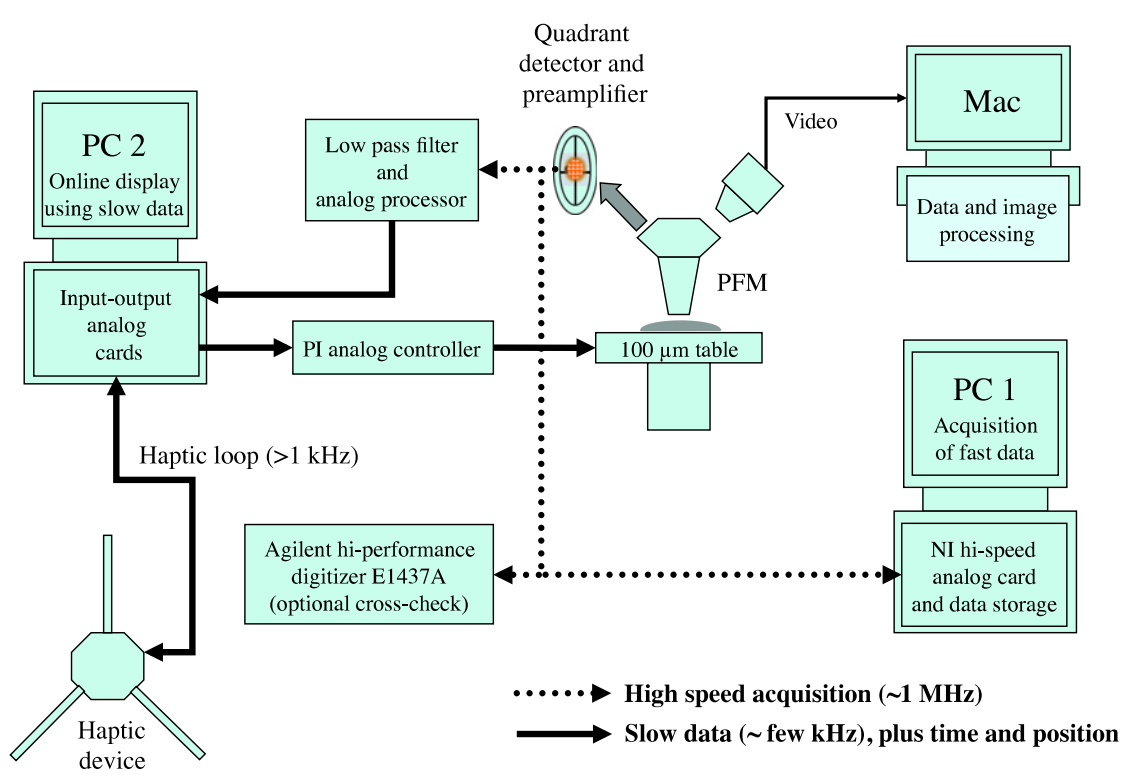

Figure 4. Set-up of the nanomanipulator.

(i) Home-made photonic-force microscope. Its construction details have been discussed in section 2 .

(ii) Proprietary analog module. It is used to filter and process the three PFM signals, as described in section 3.

(iii) NanoFeel 300 haptic device (model DHD-6DOF version 1.3, Force Dimension) equipped with a joystick with six degrees of freedom ${ }^{5}$.

(iv) Precise, micrometer stage and its analog controller. Manufacturer: Physik Instrumente ${ }^{6}$, stage type P-5173CL, horizontal movement $(100 \mu \mathrm{m} \times 100 \mu \mathrm{m}$, vertical $20 \mu \mathrm{m}$, accuracy (noise-level motion) $1 \mathrm{~nm}$ ); controller: E-503-00, E-509CA.

(v) Two PC systems serving as high- and low-speed data acquisition and processing units. The PCs are linked by a TCP/IP network which allows for the synchronization of the high- and low-speed acquisitions. The controlling software is distributed between the main system (PC2) and the remote client (PC1).

(vi) Macintosh computer. It is used to visualize the sample area being probed, and for video-recording tasks. To this end, we have benefited from the commercial software MediaWorks 6.2.

We have equipped the first PC system with a high-speed analog data acquisition card (four channels, up to $8 \mathrm{MHz}$ sampling rate, model PCI-6115, National Instruments ${ }^{7}$ ); therefore, we use PC1 to perform high-speed acquisition of data. Due to their large volume and throughput, we set these data aside for offline analysis of the PFM detector signals and for the derivation of the calibration coefficients $\beta_{i}$ by [13].

The second PC system is the core of the nanomanipulator and is used for online PFM low-speed data acquisition, processing, display and control of the instruments.

\footnotetext{
5 Force Dimension (http://www.forcedimension.com/)

6 Physik Instrumente (http://www.physikinstrumente.com/)

7 National Instruments (http://www.ni.com/)
}

particular, it provides the haptic loop for the nanomanipulator joystick and controls the stage movements. Due to its importance, we now discuss its set-up in more detail.

\subsection{Haptic loop}

4.2.1. Input/output. On the one hand, the haptic loop is driven by the six data-related signals that correspond to the six entries $\left(s_{11}, s_{22}, s_{33}, s_{12}, s_{23}, s_{31}\right)$ of the scatter matrix $\mathbf{S}$. These signals are available at the output of the analog processor shown in figure 3 . We sample them at a low frequency rate using low-cost analog input/output cards (PD2-MF-16150/16H and PD2-AO-8/16, United Electronic Industries, Inc. $^{8}$ ). For monitoring purposes, we also sample a low-pass version of the total intensity reaching the QPD. On the other hand, the haptic loop generates three output signals to control the stage axes movements. In addition, we monitor on an external display the current refresh rate of the haptic loop. We present in figure 5 the graphical user interface (GUI) of the nanomanipulator. The software is written in Visual $\mathrm{C}++$.

4.2.2. Processes. Once launched, the system enters the haptic loop and takes advantage of TCP/IP to switch on the highspeed acquisition of data on PC1. Using the joystick, the user can immediately manipulate the sample stage, but feedback forces are exerted only after their explicit activation by the user. Several other working modes and tuning capabilities are available, such as controlling the low and high acquisition rates, switching the haptic loop on or off, and initiating the storage of high-speed data, among others. We found that switching off the haptic loop while keeping the processing and recording of signals was a particularly useful mode of operation for optical adjustment of the PFM.

After having been fully initialized, the haptic loop performs the following operations, in the given order:

\footnotetext{
8 United Electronic Industries (http://www.ueidaq.com/)
} 


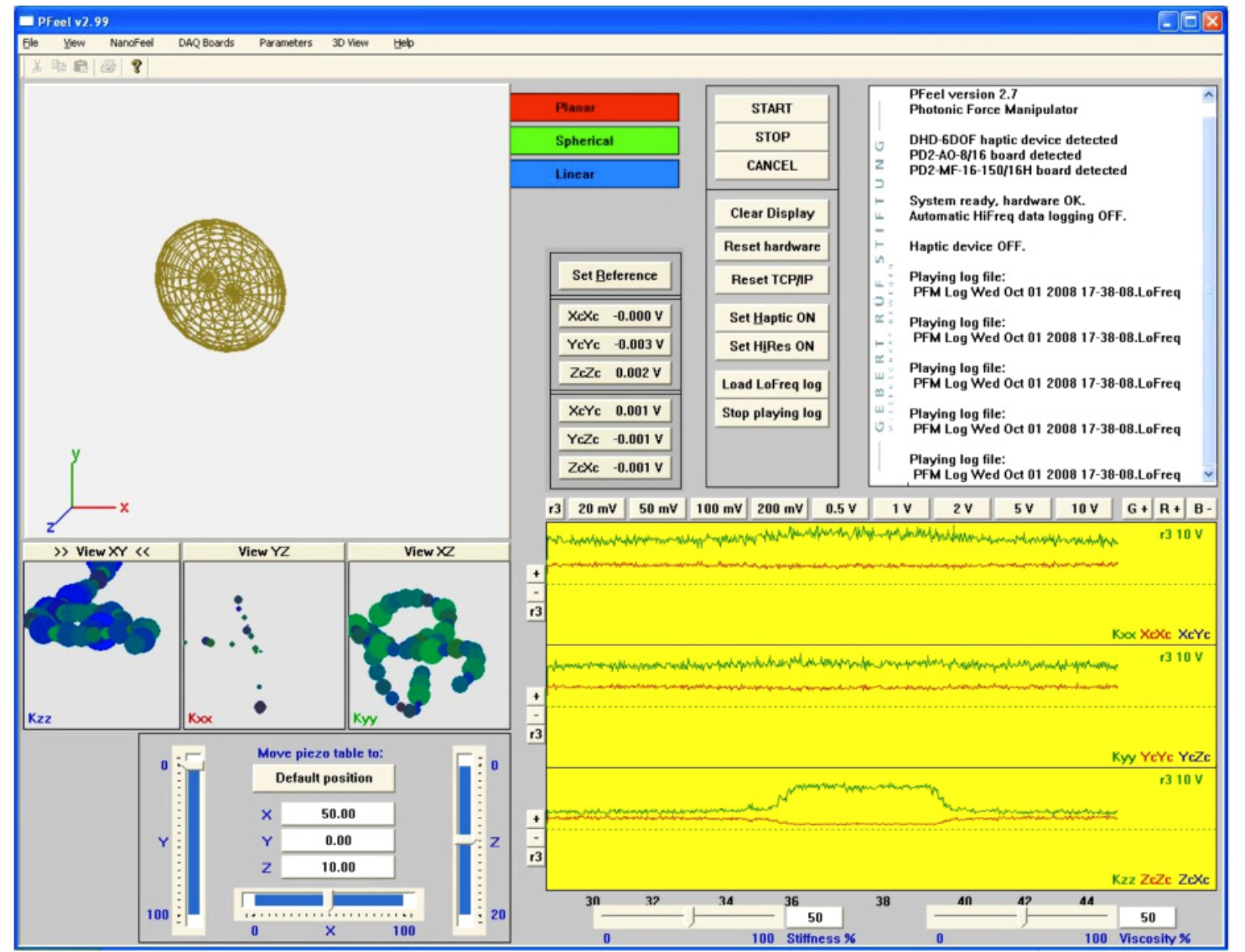

Figure 5. Graphical user interface of the nanomanipulator.

(i) Readout of the current position of the joystick. The coordinates obtained are transformed into the desired stage position.

(ii) Moving the table to the desired position. This requires the computation of the analog voltages needed for the stage controller and activation of the DAC outputs.

(iii) Readout of every output of the analog processor. This provides numerical access to the elements of the scatter matrix that was computed by our analog hardware.

(iv) Processing of the scatter matrix. This involves determining the local stiffness and computing the feedback forces to be applied to the haptic joystick. The calculations take into account the viscous (friction) and stiffness fraction of the resulting forces. In order to protect those against sudden large variations, we smooth the input data.

(v) Application of forces and torques by way of the haptic device. These operations are particularly time-consuming and actually determine the duration of a typical cycle of the haptic loop.

(vi) Visualization tasks. This involves the display of selected signals and stiffness traces, along with the $3 \mathrm{D}$ rendering of the volume accessible to the bead motion. To save on processor time, the visualization task is performed every $N$ th loop only, usually with $N=100$.

(vii) Storage in a log file of all data relevant to the current loop.
4.2.3. Display. We dedicate several windows of the GUI to the real-time display of various relevant signals. By default, all outputs from the analog processor and the calculated online diagonal stiffness matrix elements are included, while a 3D representation of the vibration of the bead is displayed in a separate window where the position of a wireframe ball reflects the position of the sample stage, while its shape and color reflect characteristics of the PFM signal.

Representing the movements of the bead is rather straightforward. However, because we have retained its offdiagonal elements, we need to pay special attention to the representation of the stiffness matrix. (We still provide a classical trace of its three main diagonal elements in the three lower-right windows of the GUI.) This is particularly true for biological matter that is organized at a length scale comparable to the bead dimensions, as this may result in a strongly anisotropic stiffness matrix since the stiffness of the material surrounding the bead may depend on the direction. We have thus provided one possible representation of the stiffness matrix as an ellipsoid that is characterized by three orthogonal axes, whose directions correspond to the three eigenvectors of $\mathbf{K}$, and whose lengths are related to its eigenvalues $\lambda_{1}>\lambda_{2}>$ $\lambda_{3}$.

Several descriptors can be used to portray directly the degree of anisotropy. The most useful and popular ones describe the dominant features of the ellipsoid as being close to a sphere (isotropic behavior, all eigenvalues have about the 
same magnitude), to a pill ('flattened' sphere, planar case, two similar eigenvalues are significantly larger than the third one) or to an elongated shape (linear case, one dominant eigenvalue) [14]. Rather than directly report the eigenvalues, which would have cluttered the display, we have instead chosen to portray the stiffness information in the color of the displayed ellipsoid. We define the index of sphericity $c_{\mathrm{S}}$, of planarity $c_{\mathrm{P}}$ and of elongation $c_{\mathrm{L}}$ as

$$
\begin{aligned}
c_{\mathrm{S}} & =\frac{3 \lambda_{3}}{\lambda_{1}+\lambda_{2}+\lambda_{3}} \\
c_{\mathrm{P}} & =\frac{\lambda_{2}-\lambda_{3}}{\lambda_{1}+\lambda_{2}+\lambda_{3}} \\
c_{\mathrm{L}} & =\frac{\lambda_{1}-\lambda_{2}}{\lambda_{1}+\lambda_{2}+\lambda_{3}} .
\end{aligned}
$$

Since we have that $c_{\mathrm{S}}+c_{\mathrm{P}}+c_{\mathrm{L}}=1$, it is easy to attribute RGB colors to these factors and to use the corresponding RGB combination to draw the ellipsoid. This results in an intuitive and uncluttered visualization that reinforces the perception of the dominant characteristics of the acquired signal. Meanwhile, a user may calibrate or disable any of the signals controlled or measured by the system. In doing so, it is possible to focus human attention on just a few of the interactions with the sample under investigation, or to exclude one or more channels from the system. This task may be easily accomplished with the use of a set-up dialog.

4.2.4. Refresh rate. The refresh rate of the loop depends on the hardware and software operations that need to be performed. We observe that the computationally most demanding component is related to the haptic device itself. In the current set-up, an off-the-shelf PC dealing with feedback forces alone would perform with a refresh rate of about $3 \mathrm{kHz}$. However, when the determination of feedback torques is also taken into consideration by the haptic loop, the refresh rate drops to about $1.2 \mathrm{kHz}$. The performance is decreased even further by reason of the general system load due to the calculations and operations that involve the GUI display (e.g. $3 \mathrm{D}$ rendering, running trace of $\left.\left(s_{11}, s_{22}, s_{33}\right)\right)$. Fortunately, the refresh rate of the haptic loop always meets or exceeds $1.15 \mathrm{kHz}$, which is sufficient for human perception. Therefore, no special measures like the use of software threads or the setting of process priorities are required.

In addition to high-speed data, our software retains in an accompanying file a list of records, with one record per loop cycle. The stored information consists of a time stamp, stage position, raw analog-processor signals and also a few selected derived values, for example the components of the stiffness matrix. A user may then load back this file into a PFeel program or import it into a spreadsheet for further processing.

\section{Preliminary results}

We have examined the response of our system under two different conditions: in free water, and while approaching a glass surface. We have performed these experiments with plastic beads of various diameters.

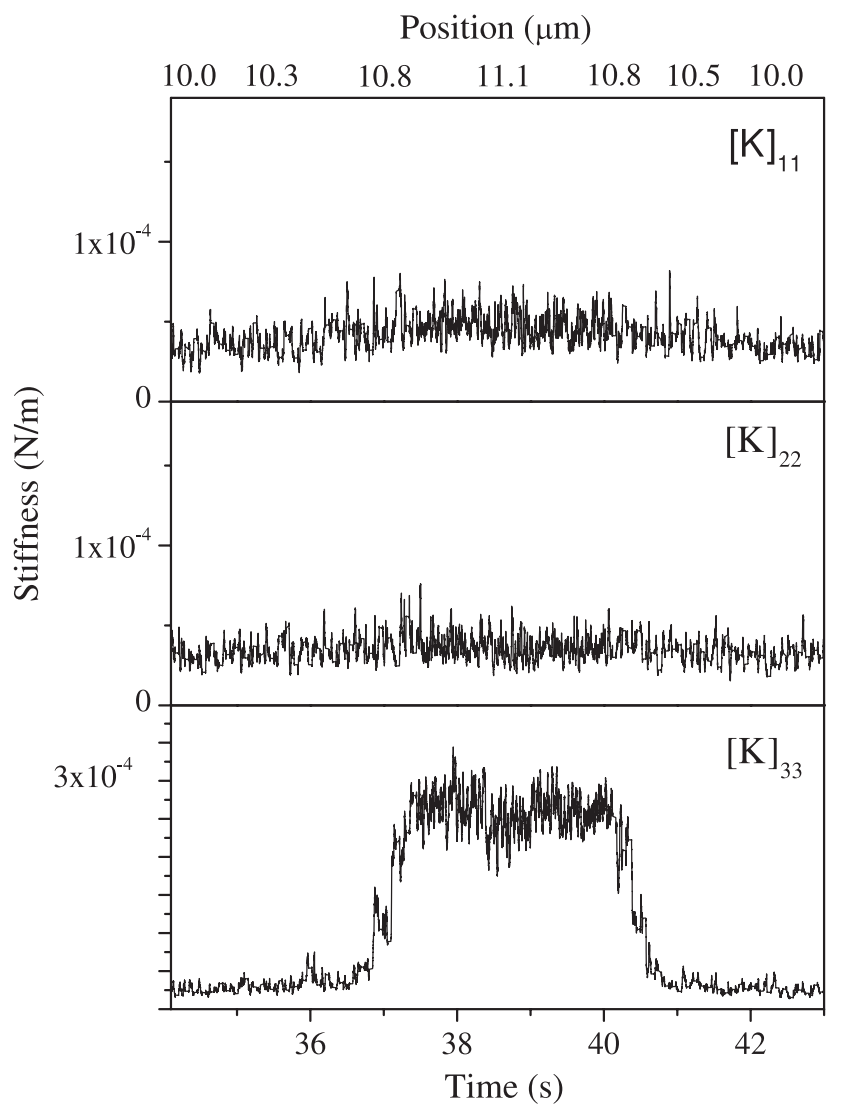

Figure 6. Main components of the trap stiffness while approaching a glass surface and then going away. (The trap positions are provided in $\mu \mathrm{m}$ on the top of the graph.)

We present in figure 6 an example of stiffness computed according to (4). It shows the three main components of $\mathbf{K}$ during a motion of the trap that was perpendicular to a glass/water interface (first approaching it, then moving away). The component $[\mathbf{K}]_{33}$ of the stiffness matrix corresponds to the axis that is perpendicular to the approached surface. We observe that it experienced a strong increase during the last micron of the approach.

We have compared the stiffness resulting from (4) to the stiffness computed offline by the so-called 'corner frequency' method applied to the recorded high-speed data [15]. We illustrate in figure 7 the linear dependence of the results obtained with the two methods; we observe that different laser powers and bead sizes result in significantly different trapping forces; yet, the classical method and the real-time method proposed in this paper produce essentially identical results.

We compare in figure 8 the value of $\tilde{k}_{1}$ as estimated by the traditional processing of the high-speed data, which we write $\left[\mathbf{K}_{\mathrm{H}}\right]_{11}$, against the values estimated by our proposed system using the low-speed data resulting from the analog hardware of section 3, which we write $\left[\mathbf{K}_{\mathrm{L}}\right]_{11}$. To get a feel for the statistical variability of the measurements, we have performed them in different samples of pure water. We observe that $\left[\mathbf{K}_{\mathrm{L}}\right]_{11}$ tends to be slightly larger than $\left[\mathbf{K}_{\mathrm{H}}\right]_{11}$. This seems to be related to the level of noise since, when experimenting with small beads - where the signal-to-noise ratio is lowerthe discrepancy between $\left[\mathbf{K}_{\mathrm{H}}\right]_{11}$ and $\left[\mathbf{K}_{\mathrm{L}}\right]_{11}$ becomes larger. 


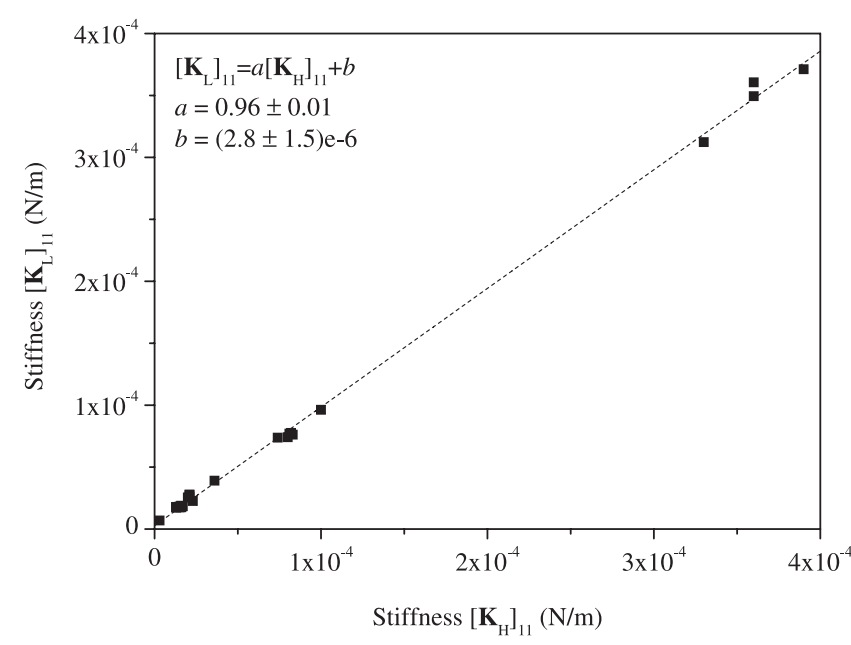

Figure 7. Match between stiffness values obtained with low- (L) and high-speed $(\mathrm{H})$ acquisitions.

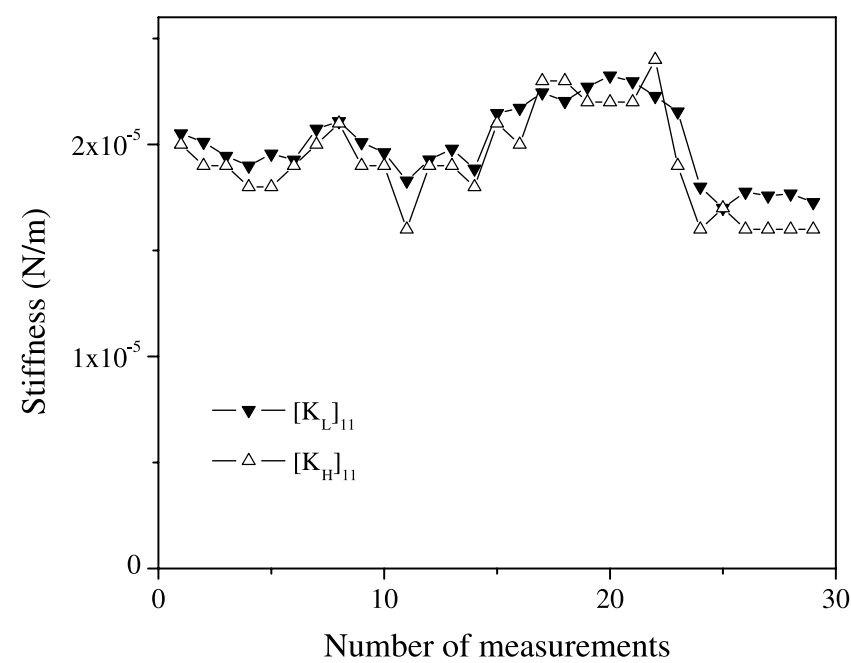

Figure 8. Stiffness $\left[\mathbf{K}_{\mathrm{L}}\right]_{11}$ and $\left[\mathbf{K}_{\mathrm{H}}\right]_{11}$ of the trap in free water obtained from the low- $(\mathrm{L})$ and high-speed $(\mathrm{H})$ acquisitions with $0.53 \mu \mathrm{m}$ diameter beads in different samples.

We show in figure 9 the dependence of the trap stiffness on the position of the trap while approaching a glass surface. The lower graph corresponds to the measurement presented in figure 6. We recognize the sinusoidal modulation of the stiffness near the surface of the liquid cell. The period of modulation is $d=\lambda /(2 n)$, where $\lambda$ is the laser wavelength and $n$ the refraction index of water. We have measured $d=450 \pm 50 \mathrm{~nm}$, which is compatible with the explanation proposed by $[12,16]$ as a result of the standing light wave between the bead and the surface. In our case, however, the amplitude of this modulation has increased for smaller beads, contrary to the observations in [16].

\section{Conclusion and outlook}

We have designed a versatile single-beam optical-tweezers system capable of measuring force and stiffness in real time and in three dimensions. The system, equipped with a haptic $3 \mathrm{D} / 6 \mathrm{D}$ joystick, allows the user to interactively move the bead

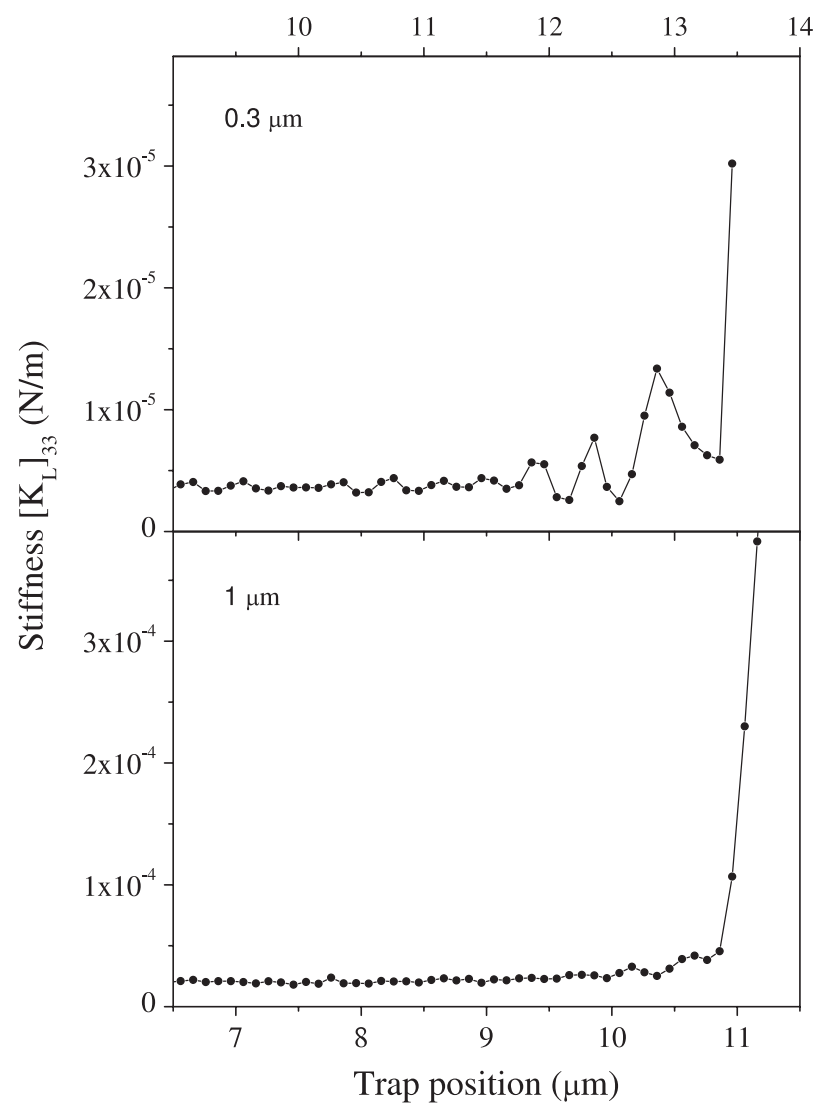

Figure 9. Stiffness $\left[\mathbf{K}_{\mathrm{L}}\right]_{33}$ of the trap in function of the trap position while approaching a glass surface. The upper graph corresponds to a bead diameter of $0.3 \mu \mathrm{m}$, while the lower graph corresponds to a bead diameter of $1 \mu \mathrm{m}$.

and obtain the stiffness matrix of a liquid or biological sample with high spatial resolution (a few $\mathrm{nm}$ ) and update frequency of $1 \mathrm{kHz}$.

Our system is targeted to the real-time measurements of intracellular properties such as stiffness or viscoelastic moduli. While making the analysis quantitative requires the calibration of the signals with the help of high-speed data at the beginning of an experiment, relative changes of the stiffness are displayed, measured and felt by the operator interactively through the haptic joystick.

We were able to successfully apply our nanomanipulator to an intracellular optical-trapping task. The results of the biological measurements will be published elsewhere, along with the technical derivations that lead to (4).

\section{Acknowledgment}

This work has been financed by 'GEBERT-RÜF STIFTUNG,' Project GRS-012/06.

\section{References}

[1] Pralle A, Florin E-L, Stelzer E H K and Hörber J K H 1998 Local viscosity probed by photonic force microscopy Appl. Phys. A 66 S71-3 
[2] Tolić-Nørrelykke I M, Munteanu E-L, Thon G, Oddershede L and Berg-Sørensen K 2004 Anomalous diffusion in living yeast cells Phys. Rev. Lett. 93078102

[3] Guzmán C, Flyvbjerg H, Köszali R, Ecoffet C, Forró L and Jeney S 2008 In situ viscometry by optical trapping interferometry Appl. Phys. Lett. 93184102

[4] Jeney S, Stelzer E H K, Grubmüller H and Florin E-L 2004 Mechanical properties of single motor molecules studied by three-dimensional thermal force probing in optical tweezers ChemPhysChem 5 1150-8

[5] Tischer C, Altmann S, Fišinger S, Heinrich Hörber J K, Stelzer E H K and Florin E-L 2001 Three-dimensional thermal noise imaging Appl. Phys. Lett. 793878

[6] Lukić B, Jeney S, Sviben Ž, Kulik A J, Florin E-L and Forró L 2007 Motion of a colloidal particle in an optical trap Phys. Rev. E 76011112

[7] Jeney S, Lukić B, Kraus J A, Franosch T and Forró L 2008 Anisotropic memory effects in confined colloidal diffusion Phys. Rev. Lett. 100240604

[8] Rohrbach A, Tischer C, Neumayer D, Florin E-L and Stelzer E H K 2004 Trapping and tracking a local probe with a photonic force microscope Rev. Sci. Instrum. 75 2197-210

[9] Gittes F and Schmidt C F 1998 Interference model for back-focal-plane displacement detection in optical tweezers Opt. Lett. 23 7-9
[10] Rohrbach A and Stelzer E H K 2002 Three-dimensional position detection of optically trapped dielectric particles J. Appl. Phys. 91 5474-88

[11] Wang M C and Uhlenbeck G E 1945 On the theory of the Brownian motion II Rev. Mod. Phys. 17 323-42

[12] Neuman K C and Block S M 2004 Optical trapping Rev. Sci. Instrum. 75 2787-809

[13] Allersma M W, Gittes F, deCastro M J, Stewart R J and Schmidt C F 1998 Two-dimensional tracking of NCD motility by back focal plane interferometry Biophys. $J$. 74 1074-85

[14] Zhang S and Laidlaw D H 2004 Diffusion tensor MRI visualization The Visualization Handbook ed C D Hansen and C R Johnson (New York: Academic) chapter 16 pp 327-40

[15] Berg-Sørensen K, Peterman E J G, Weber T, Schmidt C F and Flyvbjerg H 2006 Power spectrum analysis for optical tweezers. II: laser wavelength dependence of parasitic filtering, and how to achieve high bandwidth Rev. Sci. Instrum. 7763106

[16] Schäffer E, Nørrelykke S F and Howard J 2007 Surface forces and drag coefficients of microspheres near a plane surface measured with optical tweezers Langmuir 23 3654-65 